\title{
Design, and participant enrollment, of a randomized controlled trial evaluating effectiveness and cost-effectiveness of a community-based case management intervention, for patients suffering from COPD
}

This article was published in the following Dove Press journal:

Open Access Journal of Clinical Trials

18 June 2015

Number of times this article has been viewed

\author{
Sabrina Storgaard \\ Sørensen' \\ Kjeld Møller Pedersen' \\ Ulla Møller Weinreich ${ }^{2,3}$ \\ Lars Holger Ehlers' \\ 'Danish Center for Healthcare \\ Improvements, Faculty of Social \\ Sciences and Faculty of Health \\ Sciences, Aalborg University, Aalborg \\ East, Denmark; ${ }^{2}$ Department of \\ Respiratory Medicine, Aalborg \\ University Hospital, Aalborg, \\ Denmark; ${ }^{3}$ The Clinical Institute, \\ Aalborg University Hospital, \\ Aalborg, Denmark
}

Background: Case management interventions are recommended to improve quality of care and reduce costs in chronic care, but further evidence on effectiveness and cost-effectiveness is needed. The objective of this study is the reporting of the design and participant enrollment of a randomized controlled trial, conducted to evaluate the effectiveness and cost-effectiveness of a community-based case management model for patients suffering from chronic obstructive pulmonary disease (COPD). With a focus on support for self-care and care coordination, the intervention was hypothesized to result in a reduced number of COPD-related hospital admissions

Patients and methods: The design was a randomized controlled trial conducted from 2012 to 2014 with randomization and intervention at patient level. The study took place in Aalborg Municipality, a larger municipality in Denmark. A total of 150 COPD patients were randomized into two groups: the case-managed group and the usual-care group. Participant characteristics were obtained at baseline, and measures on effectiveness and costs were obtained through questionnaires and registries within a 12-month follow-up period. In the forthcoming analysis, effectiveness will be evaluated on COPD-related hospital admissions, mortality, health- related quality of life, and self-care. An economic evaluation will examine the cost-effectiveness of case management against current usual care from the perspective of the health care sector.

Results: Baseline characteristics were comparable between the two groups except for the prevalence of cancer, which were borderline significantly higher in the case-managed group. This baseline difference will be adjusted for in the final analysis. A very low dropout rate for the intervention was observed, indicating that the intervention was well accepted.

Conclusion: Presented in a forthcoming paper, the results of the study will provide new evidence to the future organization of case management

Keywords: personalized care, disease management, health-related quality of life, costeffectiveness analysis

\section{Introduction}

Chronic obstructive pulmonary disease (COPD) is a burdensome disease imposing a significant disability and impaired quality of life for the patients as well as generating high health care costs for the society. The disease is irreversible and treatments are directed toward management of the disease to prevent its further progression. ${ }^{1}$ 
Development of new disease management strategies for chronic diseases has been prioritized in recent years, and the term "case management" has received much focus. Case management involves central elements of the Chronic Care Model, ${ }^{2}$ and the concept is believed to be an effective and efficient approach to manage patients with chronic diseases and complex health care needs. ${ }^{3}$ No clear definition of case management exists; however, the term covers a broad range of interventions with a patient-centered approach, where the patient is navigated through a complex health-related process by an individual case manager or a small case management team. To date, numerous studies evaluating interventions of case management for COPD exist, which differ in relation to setting, organizational approach, providers, level of coordination of health care and social services, patient selfmanagement education, etc. ${ }^{4-17}$ The substantial heterogeneity of content and methods used makes it difficult to identify the key components of an effective model. Furthermore, with the exception of a few studies, work procedures such as real time spent with patients and degree of coordination with other health care professionals, are often also not accounted for which keeps the reader in the dark as to the character and intensity of the case management intervention. . $^{511,16}$ Previous studies have, for instance, had varying caseloads of 14-250 patients per case manager, ${ }^{16,18}$ which inevitably must impact on the service provided for each patient. In light of this, the authors believe that there is a need for further and more transparent research of case management for patients suffering from COPD.

In addition, the existing studies evaluating case management for COPD patients have primarily focused on examining the effectiveness of case management. However, when evaluating new interventions it is important not only to evaluate the effectiveness but also to address the associated costs of an intervention. Despite more or less successful attempts to estimate the costs of case management for COPD,,$^{5,11,14,15,18}$ there exists no full health economic evaluation to date, and it is therefore unknown if it is a cost-effective disease management strategy for this patient group. The cost-effectiveness of health technologies might vary from place to place due to factors such as clinical practice patterns, the availability of health care resources, and unit costs. ${ }^{19}$ Cost-effectiveness can thus vary between different forms of case management and between countries. In order to heighten transferability of results to other settings, it is therefore of great importance that cost-effectiveness analyses are transparent and meticulously reported following international guidelines. ${ }^{20,21}$
The objective of this study is the reporting of the design of a randomized controlled trial (RCT), conducted to evaluate the effectiveness and cost-effectiveness of case management for patients suffering from COPD.

The intervention focuses on support for self-care and on care coordination, which is hypothesized to significantly reduce the number of COPD hospital admissions. What this article adds to the existing literature is a detailed reporting of the RCT design and the used methods, hereunder, the specific content and characteristics of the intervention. This will allow readers to properly evaluate relevance and transferability of the used methods. Additionally, in a forthcoming paper a full health economic evaluation of case management for COPC, consisting of a transparent cost-utility analysis (CUA) will be undertaken. Once results are published, this will enable readers to determine whether the health technology will be relevant in their setting.

\section{Materials and methods \\ Study design and setting}

The study was conducted as a single-center, parallel-group RCT, where participants assigned to the control group received current standard care, and participants in the case-managed group received case management in addition to current standard care. The study took part in Aalborg Municipality, a larger municipality in the North Region of Denmark with approximately 200,000 inhabitants. The study was conducted in the period between March 2012 and January 2014 with ongoing enrollment of participants through the first 9 months. Each participant was followed for 12 months.

A RCT in combination with a qualitative component is regarded as the research design best suited for answering questions regarding effectiveness and mechanisms of action. ${ }^{22} \mathrm{On}$ these grounds, interviews were conducted with the case manager throughout the study. These will be evaluated in a future paper to assess work procedures, usability, and feasibility of case management for COPD patients in a Danish setting.

The study was conducted in accordance with the Helsinki Declaration. The study was granted an exemption from requiring ethics approval from the Danish Ethical Committee for Medical Research in the North Denmark Region, but applied for and received data approval from the Danish Data Protection Agency in order to register and process personal data obtained during the study.

\section{Participants}

Participants were eligible for the study, if they had been referred by their general practitioner or respiratory specialist to a pulmonary rehabilitation course at the local rehabilitation 
center in Aalborg in the year 2011, irrespective of whether the participants had completed the course or not. All COPD patients in Aalborg Municipality, entitled for rehabilitation, are referred to the local rehabilitation center. In accordance with national ${ }^{23}$ and international ${ }^{1}$ guidelines, COPD patients in Denmark should be referred to rehabilitation when presenting with a modified medical research council score (mMRC) $\geq 2$, and can be referred to rehabilitation if presenting with a $\mathrm{mMRC} \leq 1$. Participants were excluded from the study if they were participating in other research studies, or if they declined to give their written consent for participation. All participants were to live in Aalborg Municipality and therefore had a maximum travelling distance of $40 \mathrm{~km}$ to the case manager office.

Eligible patients were invited by letter to take part in the study. In case the patient agreed to participate, an introductory meeting was arranged within the next 14 days where written informed consent was obtained from all participants.

\section{Choice of case manager}

The case manager had more than 20 years of experience as a COPD nurse, and had worked at both inpatient and outpatient pulmonary hospital clinics and had most recently worked as a COPD rehabilitation nurse. The case manager had previously completed several courses on motivational dialogue, and had, before initiation of the study, completed a health and lifestyle-coaching course to optimize her coaching skills.

\section{Randomization procedure}

Randomization lists were generated on the basis of random, permuted blocks of five to ensure approximate balance over time. At the introductory meeting the participants would draw a sealed envelope, containing a random number. This number would correspond to a number on the randomization lists that would indicate whether the participant was assigned to the case-managed group or the usual-care group. The randomization list was stored and handled by an impartial clinical researcher not involved in the study. Following the meeting, the participant would be contacted and informed about which group he or she had been assigned to.

\section{Control: the usual-care group}

In Denmark, all registered residents are entitled to free and equal health care. The usual-care group received current standard care as according to most recent evidence-based guidelines, ${ }^{1}$ and would not have contact with the case manager during the following 12 months.

\section{Intervention: the case-managed group}

In addition to usual care, participants randomized to the intervention received case management consisting of the following activities.

\section{Assessment of health risk and care planning}

Shortly following randomization, a start-up meeting was arranged for the participants assigned to the case-managed group. Preferably, the meeting took place at the case manager office; however, the meeting could take place in the participant's own home, depending on impact of disease or in case of concerns about caregiver capability. Potential problem areas were identified, and the obtained information was used to develop a care plan that addressed the identified needs and problems. Hence, the care plan was based on what was important for each individual participant, and was continuously revised throughout the study. Involvement of caregivers and family members in this was optional. Following the meeting, the case manager informed the participant's general practitioner and potential home health carers/nurses about the participant's participation in the study, and, upon agreement with the participant, they were informed that they could contact the case manager regarding the participant at any time during the study period.

\section{Monitoring of individual health status}

The case manager was able to schedule follow-ups according to individual participant needs; hence resulting in some participants receiving more intensive case management than others. As a minimum, each participant would receive a follow-up telephone call once every month as well as a follow-up meeting at the case manager's office or in the participant's own home every third month in order to ensure prompt detection of potential problems. At each follow-up, the case manager would ask about the participant's general health status in order to identify signs of exacerbations. Topics that were to be addressed at follow-up would depend on the individual participant, but could, for example, concern medication adherence and weight gain/loss, etc. Furthermore, the participants were encouraged to contact the case manager if they had any questions relating to their disease or medical care, in case of worsening of symptoms, and after a hospital admission or emergency room visit. However, the case manager was not to have any direct role in chronic disease treatment, and was not to take over the role and responsibility of other health care providers such as the participant's general practitioner, etc. 


\section{Promotion of self-care and autonomy}

In order to strengthen the participant's abilities to provide self-care and support their autonomy, the case manager sought out and listened to the participant's perspectives through motivational dialogue, providing choices rather than ultimatums, and providing appropriate challenges and celebrating successes, as well as giving positive performance feedback. Attention was paid not to generate needs that were not present at the beginning of the intervention.

\section{Provision of COPD knowledge and support}

Providing knowledge of COPD, associated consequences, and management hereof was a requirement for the participants to take on self-care tasks successfully. This component of the intervention was based on motivational dialogue with the case manager and was tailored for each participant. The component included:

- Introduction to the anatomy and physiology of the lungs and the impact of COPD hereupon.

- Explanation of obtained spirometry outcome to the participant.

- Instruction and control of correct inhalation and coughing technique.

- Assessment of pharmacological treatment, and a dialogue on why, when, and how to take the medication correctly.

- Instructions on how to prevent, detect, and deal with acute exacerbations, including counseling on how to prevent infections and advice on when to contact the general practitioner.

- Conversation about how to incorporate physical activities and exercise in daily life.

- Dietary advice for under-/overweight participants.

- Dialogue on the consequences of smoking for current smokers and advice on smoking cessation for those interested herein.

- Patient preparation of appointments through the provision of questions and issues to take up with physicians.

- Psychosocial counseling and support.

Relevant handouts were distributed when needed, which were tailored to the needs of the individual participant or caregiver.

\section{Facilitation of relevant health and social services and coordination of care}

The case manager introduced the participants to relevant health and social services and eased their access to the services, eg, through assistance in how to apply for the services. The case manager encouraged the participants' participation in health activities, follow-up visits, etc., and provided reminders for participants with appointment adherence difficulties. Generally, the case manager's function was to make the participants equipped to take action themselves; however, if the participants did not have the resources to do so, the case manager took on a more active role and coordinated care for the participant. The case manager cooperated with, but did not take over the responsibility of other health care providers. Instead, the case manager was an addition to usual care.

\section{Outcome measures}

The primary outcome for the study was a change in rate of COPD hospitalizations within the 12-month follow-up period, as it was hypothesized that the provision of case management can reduce the rate of COPD-related hospital admissions. Secondary outcome measures were: all-cause mortality, health-related quality of life (HRQoL), selfcare, and cost-utility of case management - all within the 12-month follow-up period. The clinical impact of case management was assessed as being relevant if the mortality was unchanged or lowered and if HRQoL was increased and self-care abilities improved. Cost-effectiveness of case management was evaluated by use of a CUA. The incremental cost-effectiveness ratio was therefore calculated as the cost per quality-adjusted life year (QALY). All outcomes were measured at individual level.

\section{Data and data collection}

\section{Effects}

Data on HRQoL were obtained through use of the generic questionnaires SF-12 and EuroQol 5-dimensions 3-level (EQ-5D-3L). The EQ-5D-3L questionnaire was applied to calculate QALY used in the CUA, and Danish societal weights were applied for calculating utility scores. ${ }^{24}$ The disease-specific questionnaire St George's Respiratory Questionnaire (SGRQ) was used to obtain a measure of overall health, daily life, and perceived well-being, whereas the Patient Activation Measure (PAM-13) questionnaire was used to measure change in self-care within the 12 months period. Since individual participant factors may influence the outcome of the intervention, a questionnaire covering demographic, disease-specific, and psychosocial topics was developed and handed out to the participants. The questionnaires were administered and fulfilled at the introductory meeting and again after 12 months at a finale meeting. The participants were to fill out the questionnaires themselves, but 
a researcher was present at both occasions in matters of doubt. At both meetings, a spirometry was performed as well.

\section{Costs}

Data for the CUA were gathered alongside the trial. Costs were considered from the perspective of the health care sector, meaning that all costs to health care providers involved in treating, caring for, and monitoring the participants were included. Adopting a health care sector perspective means that the total cost excludes the cost incurred by the participants and their relatives, eg, transportation to and from the case manager's office and loss of time, as well as costs due to productivity loss to society. The health care sector perspective is widely used in health economic evaluations and is especially adopted in CUA, since including costs due to productivity loss in such an analysis poses a risk of double counting. ${ }^{25}$

The costs included in the analysis were direct diseaserelated costs in the primary health care sector (general practitioner contacts, physiotherapy, etc), the secondary health care sector (in- and outpatient hospital treatment and emergency room visits), cost for community care (home nurse care, nursing homes, household cleaning, and training), costs for prescription medication, and intervention costs (case manager salary, cost for coaching course, and cost for driving in case of home visits, etc). All Danish citizens have their own unique ten-digit identification numbers, which are used for registration every time a citizen contacts the health care sector, and data are registered in various registers. In this study, register data for each participant were extracted by use of the identification number. Data on health care usage in the primary and secondary health care sector and prescription medicine were extracted from the regional register of North Denmark:, Health, Planning, and Quality department. The register holds data on all contacts between patients and the primary and secondary health care sector and sales of prescription medicine for all patients living in and receiving medical care within the North Denmark Region. For primary health care service use, the register contains information on the date the service was received, type of service, and the reimbursement fee paid by the National Health Insurance to the health care provider. General practitioners are paid by a mixture of capitation and fee for service, which will be accounted for in the analysis. The use of secondary health care is registered in accordance with the Danish diagnosisrelated grouping (DRG) system, which enables extracting of information on number of admissions, length of stay, number of outpatient visits, and emergency room visits, and all data are linked to a DRG system. The associated DRG charges will be used as unit costs for secondary care. The DRG charges reflect the average costs for treating patients with similar conditions and processes at a Danish hospital, and are used for reimbursement between regional health care authorities providing hospital treatment if a patient from one region is treated at a hospital in another region. The registered data on the participants' use of prescription medicine are based on transaction reports from the dispensing pharmacies, and holds information on the date the receipt was presented at the pharmacy, the package identifier, and the number of packages issued. Data on the use of community care was collected from the municipal register of Aalborg Municipality.

Costs related to trial execution, such as ongoing evaluation meetings with the research staff and the case manager to ensure protocol adherence throughout the trial, were not incorporated into the economic evaluation.

\section{Other}

In order to shed light on and evaluate the intensity and character of the intervention, the real time spent by the case manager with each participant, and the use of home visits and telephone calls were registered via premade schemes that the case manager was to fill in after each contact with a participant. The degree of coordination with other health care providers involved in the treatment of the individual participant was registered as well.

These data were combined with semi-structured face-toface interviews held with the case manager at 6 and 12 months after study initiation. The purpose of the interviews was to uncover work procedures, intervention strengths and weaknesses, suggestions for improvement, components that were effective/not effective, and experienced obstacles when providing case management in order to assess the usability and feasibility hereof in a Danish setting. The interviews were held by the same researcher and were digitally recorded. Verbal informed consent was obtained from the case manager before initiation of the interviews.

\section{Sample size calculations}

It was calculated that 63 participants were required to have an $80 \%$ chance of detecting a reduction in hospital admissions of 0.6 per patient per year using a two-sided $P$-value of 0.05 (smallest detected difference) ${ }^{12}$ in both groups. However, with an expected loss to follow-up of $20 \%$, the total required sample size was estimated to be approximately 75 participants in both groups. 


\section{Statistical analysis}

A statistical analysis plan was developed prior to study initiation, and the plan will be used as basis for the impending final statistical analysis.

Baseline characteristics for the two groups will be analyzed using descriptive statistics such as Student's $t$-test for continuous variables and chi-squared tests for categorical data. For continuous variables, normally distributed data will be presented as mean $( \pm \mathrm{SD})$, whereas non-normal distributed data will be presented as medians (quartiles). Categorical variables will be presented as numbers (\%). The associated $P$-value will be presented for all variables, and the level of significance will be set at $P<0.05$.

The impending statistical analysis will be performed according to the principle of intention to treat both in analysis of the effectiveness and cost-effectiveness of case management. ${ }^{21}$ Effectiveness of the intervention will be analyzed and presented in agreement with the Consolidated Standards of Reporting Trials (CONSORT) statement for parallel group RCT, and for pragmatic trials, ${ }^{26,27}$ whereas cost-effectiveness will be analyzed and presented in accordance with the Consolidated Health Economic Evaluation Reporting Standards (CHEERS) statement and the International Society for Pharmacoeconomics and Outcomes Research (ISPOR) RCT-CEA task force report for good research practice for cost-effectiveness analysis alongside clinical trials. ${ }^{20,21}$

In case the randomization does not lead to sufficiently comparable baseline values between the groups, the concerning variables will be used as covariates when conducting univariate and multivariate analysis of effects and costs. Missing data is to be expected when conducting clinical and health economic research. Data obtained from the registers are expected to be close to complete, due to the rigorous and standardized registration of health service activities in the Danish primary and secondary health care sector, whereas data obtained from the questionnaires are expected to be less complete. An evaluation of severity of missing data and specific missing data patterns will be performed. For incomplete data, multiple imputation will be used if data is considered to be missing at random or missing completely at random. No imputation will be performed if data are deemed to be missing not at random..$^{28}$

Effectiveness of the intervention will be evaluated as a comparison of difference in mean rate of COPD-related hospital admissions, mortality, HRQoL (SF-12 and EQ-5D-3L), overall health, daily life, and perceived well-being (SG-RQ), and self-care (PAM-13) between the case-managed group and usual-care group. As for the effect measures, COPD-related hospital admissions and the utility scores obtained from the
EQ-5D-3L, a univariate and multivariate analysis will be applied to obtain a point estimate effect measure and associated standard error for the case-managed group and for the usual-care group. Both hospitalization and EQ-5D-3L scores are continuous data, and the outcomes will be analyzed by use of linear regression models. The type of linear regression model used will depend on the distribution of data. The explanatory variables added to the regression models will be identified through univariate analysis.

When estimating costs, each resource parameter will be combined with a unit cost to obtain cost per person from baseline to 12 months follow-up. A point estimate for the average costs for the case-managed group and for the usualcare group and associated standard error will be derived from univariate and multivariate analysis.

Assessment of the cost-effectiveness will be evaluated by calculating a point estimate of the incremental cost-effectiveness ratio after 12 months follow-up. When calculating the incremental cost-effectiveness ratio, the difference in arithmetic mean costs between the case management intervention and usual care is divided by the arithmetic mean effects between the same two interventions. ${ }^{29}$ The effect measure for the CUA will be QALYs, which will be calculated by linear interpolation of utility scores obtained from the EQ-5D-3L between baseline and 12 months follow-up.

In order to assess the uncertainty surrounding the point estimates of mean costs and effects, the CUA will be subjected to one-way and probabilistic sensitivity analysis. One-way sensitivity analysis allows identification of those parameters that have the greatest influence on the result by varying one parameter at the time. Probabilistic sensitivity analysis, however, enables the reader of the analysis to get an understanding of the impact different uncertainties in parameter values can have on the probability of case management being cost-effective at different levels of willingness to pay for a gain in QALYs. ${ }^{29}$

The statistical analysis will be performed using the statistical software Stata v 12.

\section{Results}

Two hundred and thirty-nine letters were sent out to eligible participants before the necessary sample size of 150 participants was attained, making the participation rate $63 \%$. Those declining to participate reported being too healthy, too ill, having fear of registers, and by principle not participating in research projects as a reason of refusal. In total, 74 participants were randomized to the usual-care group, whereas 76 were randomized to the case-managed group. During the 12 months follow-up, four participants in the 


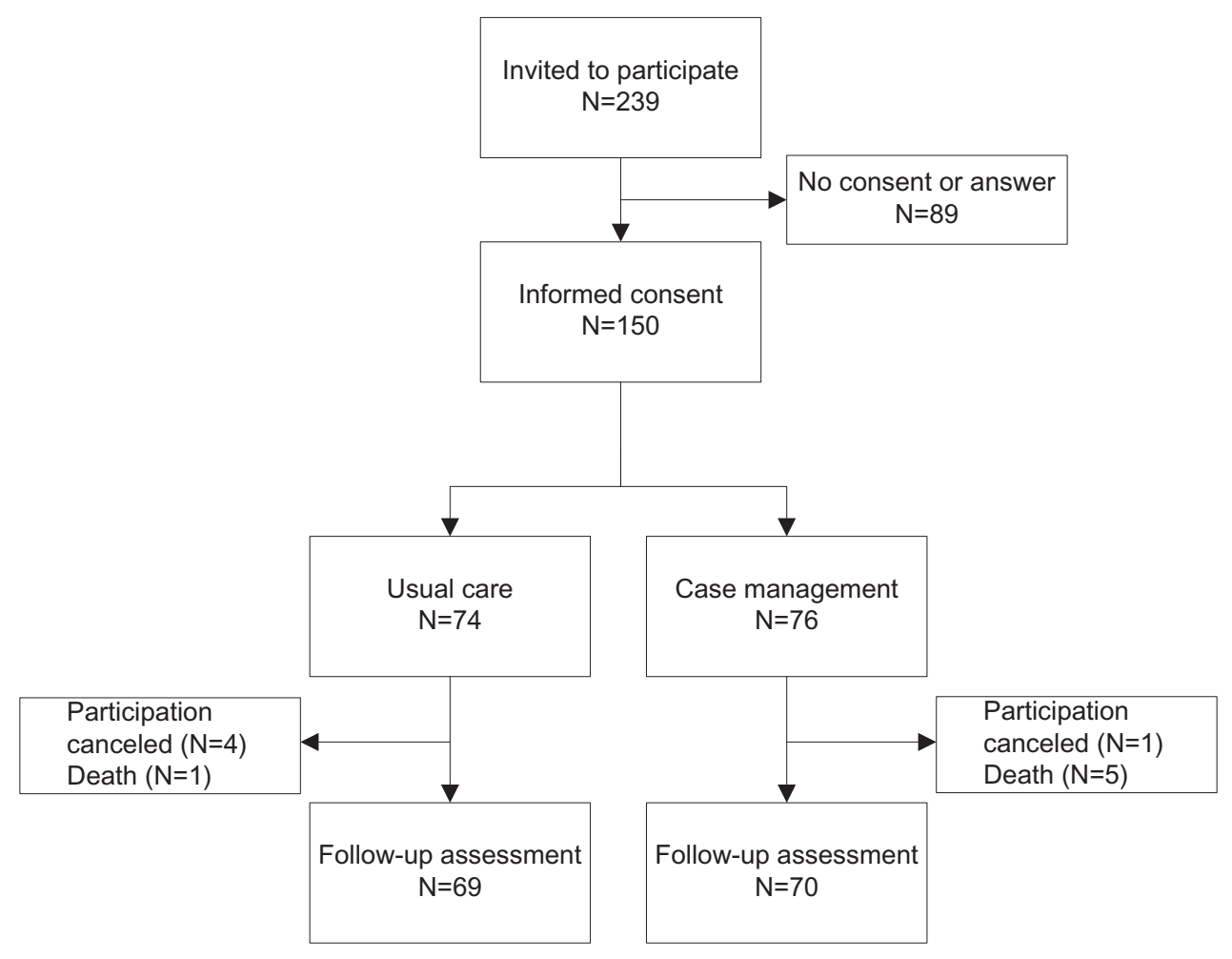

Figure I Flowchart of participants.

usual-care group dropped out of the study, whereas only one in the case-managed group dropped out. Three participants, two from the usual-care group and one from the casemanaged group, indicated the reason for dropout as flare-up of a former cancer, whereas the reason for dropout for the two others was unknown. One participant in the usual-care group and five participants in the case-managed group died during the study period. However, two of the deceased participants in the case-managed group died immediately following randomization and before receiving any case management. A flowchart illustrating the study is illustrated in Figure 1.

Table 1 shows participant baseline characteristics for the usual-care group and the case-managed group. Only minor differences can be seen between the two groups. The case-managed group comprised slightly more males than the usual-care group, and a greater percentage of the participants in the case-managed group were employed than the usualcare group, although none of the differences were statistically significant. As for cancer, a borderline significant trend was seen, with a greater share of participants in the case-managed group having or having had a diagnosis of cancer.

\section{Discussion}

This article outlines the study protocol and participant recruitment for a study evaluating the effectiveness and costeffectiveness of a community-based case management model for COPD patients in a Danish setting. Hospital admissions were chosen as the primary outcome of the study, since other care coordinating studies have been found to decrease the rate of hospital admissions., ${ }^{4,8,9,12}$ In addition, expenditures for hospital admissions have been found to account for $>70 \%$ of all COPD-related medical care costs, ${ }^{30}$ which from a costeffective approach further augments the choice of primary outcome.

The statistical power calculation found a need of approximately 75 participants in each group in order to detect a reduction in hospital admissions of 0.6 per participant per year using a two-sided $P$-value of 0.05 .

Based on evidence from a case management study by Bourbeau et al supporting caseloads of up to 70 participants, when taking both costs and effects into consideration, it was decided that it would be sufficient and optimal to affiliate only one case manager to the study. ${ }^{18}$

When conducting a study on case management, it must be decided how each participant should be followed throughout the study. Telephone calls were believed to be the cheapest method of follow-up; however, this method could potentially lack the influence of in-depth personal meetings and thereby potentially impact on effectiveness of the study. Therefore, in order to find a balance between effectiveness and costs, follow-up during the study period was chosen as a mixture of telephone calls and personal meetings. 
Table I Baseline characteristics of study participants

\begin{tabular}{|c|c|c|c|}
\hline Characteristics & $\begin{array}{l}\text { Usual-care group } \\
(\mathrm{N}=74)\end{array}$ & $\begin{array}{l}\text { Case-managed group } \\
(\mathrm{N}=76)\end{array}$ & $P$-value \\
\hline Age, years, $\pm S D$ & $69.7 \pm 8.6$ & $69.1 \pm 8.3$ & 0.66 \\
\hline Male sex, n (\%) & $27(36.5)$ & $37(48.7)$ & 0.13 \\
\hline Living alone, n (\%) & $34(46.0)$ & $29(38.2)$ & 0.33 \\
\hline \multicolumn{4}{|l|}{ Educational level, n (\%) } \\
\hline Secondary school or less & $33(44.6)$ & $32(42.1)$ & \multirow[t]{4}{*}{0.32} \\
\hline Vocational education & $26(35.1)$ & $24(31.6)$ & \\
\hline Academy profession degree & $6(8.1)$ & $3(4.0)$ & \\
\hline Bachelor's or Master's degree & $9(12.2)$ & $17(22.4)$ & \\
\hline Employed, n (\%) & $4(5.4)$ & II ( $(14.5)$ & 0.10 \\
\hline \multicolumn{4}{|c|}{ Participant self-reported chronic comorbidities, n (\%) } \\
\hline Diabetes & $9(12.2)$ & $8(10.5)$ & 0.75 \\
\hline Heart disease & $40(54.1)$ & $48(63.2)$ & 0.26 \\
\hline Osteoporosis & $22(29.7)$ & $15(19.7)$ & 0.16 \\
\hline Cancer $^{\mathrm{a}}$ & $5(6.8)$ & $13(17.1)$ & 0.07 \\
\hline Current smoker, n (\%) & $20(27.0)$ & $21(27.6)$ & 0.93 \\
\hline Pack years, $(25 \%-75 \%)$ & $4 \mathrm{I}(26.4-50)$ & $39.5(27.5-50)$ & 0.90 \\
\hline BMI, $\mathrm{kg} / \mathrm{m}^{2}(25 \%-75 \%)$ & $25.8(22.9-29.3)$ & $25.2(23.3-29.1)$ & 0.98 \\
\hline mMRC dyspnea scale, \pm SD & $2.1 \pm 0.6$ & $2.1 \pm 0.8$ & 0.81 \\
\hline $\mathrm{FEV}_{\mathrm{I}} / \mathrm{FVC}(25 \%-75 \%)$ & $55.3(43.3-66.7)$ & $53.1(43.1-62.5)$ & 0.50 \\
\hline \multicolumn{4}{|l|}{ Airflow limitation, n (\%) } \\
\hline Mild $\left(\mathrm{FEV}_{1}>80 \%\right)$ & $5(6.8)$ & $8(10.5)$ & \multirow[t]{4}{*}{0.85} \\
\hline Moderate $\left(\mathrm{FEV}_{1}, 50 \%-80 \%\right)$ & $32(43.2)$ & $30(39.5)$ & \\
\hline Severe $(\mathrm{FEV}, 30 \%-50 \%)$ & $30(40.5)$ & $30(39.5)$ & \\
\hline Very severe $\left(\mathrm{FEV}_{1}<30 \%\right)$ & $7(9.5)$ & $8(10.5)$ & \\
\hline \multicolumn{4}{|l|}{ Physical activity, $\mathrm{n}(\%)^{\mathrm{b}}$} \\
\hline Sedentary/lightly active & $13(17.6)$ & II (I4.5) & \multirow[t]{3}{*}{0.20} \\
\hline Moderately active & $57(77.0)$ & $58(76.3)$ & \\
\hline Very active & $4(5.4)$ & $7(9.2)$ & \\
\hline All-cause hospital admission previous year, $\mathrm{n}(\%)$ & $51(68.9)$ & $56(73.7)$ & 0.52 \\
\hline COPD hospital admission previous year, n (\%) & $10(13.5)$ & $13(17.1)$ & 0.54 \\
\hline
\end{tabular}

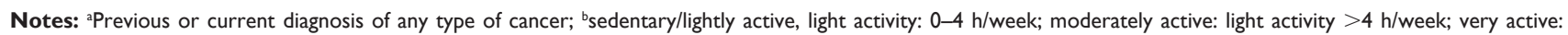
strenuous activity $2-4 \mathrm{~h} /$ week.

Abbreviations: SD, standard deviation; BMI, body mass Index; mMRC, modified Medical Research Council; FEV ${ }_{1}$, forced expiratory volume in I second; FVC, forced vital capacity.

A strength of the study is the extensive data collection. Both generic and disease-specific questionnaires were utilized, and the rigorous registration of health care usage for each participant, additionally, enables an exhaustive and nearby complete analysis of the effectiveness and cost-effectiveness of case management. This is, furthermore, believed to heighten the transferability of the cost-effectiveness analysis to other settings. Lastly, the inclusion of a qualitative component is believed to shed light on the usability and feasibility of the intervention, which could be incorporated in the final analysis.

Due to the proactive nature of the intervention, the study was chosen to take place at the community level - more specifically a local municipality. This is in contrast to the majority of studies evaluating case management for COPD patients, where case management is offered when the patient becomes so ill that they contact the hospital. ${ }^{46,10,13,14}$ Our setting was believed to be optimal in order to promote self-care and preserve autonomy, and thereby prevent deterioration of the disease, which is the pivot of the intervention. The authors believe that potential differences in outcome measures and costs will be detectable within the chosen follow-up period. However, it cannot be precluded that differences in outcome measures need a longer follow-up period in order to be detectable. In that case, access to the comprehensive Danish registries gives the opportunity to follow the participants' use of health care services over a longer time period, which could potentially be supplemented with a questionnaire survey.

A possible weakness of the study is that $37 \%$ of the eligible participants refused to participate, which could potentially influence the generalizability. The ones that refused could have poorer health or simply be too healthy to feel a need for case management. Since no baseline measures could be obtained from the participants who were unwilling to participate, it is not possible to determine to what extent the results among trial participants are generalizable to the reference population. 
The present findings indicate that baseline characteristics are comparable in the case-managed group and the usual-care group; however, for cancer a borderline significant trend was seen with a greater share of participants in the case-managed group having or having had a diagnosis of cancer. It is hypothesized that the increased mortality rate in the case-managed group at follow-up might be due to the increased prevalence of cancer within the case-managed group at baseline. This hypothesis will be sought tested in the final analysis. A very low dropout rate for the intervention was observed, indicating that the intervention was well accepted.

\section{Conclusion}

To date, studies of case management for COPD patients have focused on evaluating effectiveness, but large heterogeneity exists in contents and methods used in the studies, and often the studies suffer from incomplete reporting of work procedures, which compromises the transferability of results. Additionally, the cost-effectiveness of case management for COPD has yet to be evaluated.

This study is set to evaluate both the effectiveness and cost-effectiveness of case management for COPD. The article reports a transparent research design, which enables the reader to determine relevance of the methods used. Once results become published, it is, furthermore, believed that the comprehensive reporting based on international guidelines will support the reader in assessing if the evaluated intervention is applicable to other settings. The final analysis is expected to supply evidence for the effectiveness of case management and its associated costs, which will aid in the future organization of case management. If proven beneficial, this study will have significant health benefits for COPD patients and the health care system in general, and it is expected that the intervention can be implemented in daily routines without expensive efforts.

\section{Acknowledgments}

This research project was performed with the support from a major research grant, awarded by the North Denmark Region, Denmark. The authors would like to thank the health consultants at the unit Sundhed og Sammenhæng, North Denmark Region, Denmark, for their expertise, guidance, and feedback throughout the study period.

\section{Author contributions}

SSS led the development of the study conceptualization, design, and the refinement of study design; acquired the data, performed the baseline analysis, and interpretation of data; and drafted the manuscript for publication. LHE and KMP have been involved in the study conceptualization and development, revision of the manuscript for publication, and have provided ongoing supervision. UMW contributed with expert respiratory input for the study conceptualization and development, the revision of the manuscript for publication and with ongoing supervision. All authors have read and approved the final manuscript.

\section{Disclosure}

The authors report no conflicts of interest in this work.

\section{References}

1. Global Initiative for Chronic Obstructive Lung Disease (GOLD). Global strategy for the diagnosis, management, and prevention of chronic obstructive pulmonary disease. 2015:1-117. Available from: http:// www.goldcopd.org/. Accessed January 15, 2015.

2. Improving Chronic Illness Care (ICIC). The Chronic Care Model. Available from: http://www.improvingchroniccare.org/. Accessed January 13, 2015.

3. Schaefer J, Davis C. Case management and the chronic care model: a multidisciplinary role. Lippincott's Case Manag. 2004;9(2): 96-103.

4. Casas A, Troosters T, Garcia-Aymerich J, et al. Integrated care prevents hospitalisations for exacerbations in COPD patients. Eur Respir J. 2006;28:1130-1230

5. Leung AC, Liu C, Chow NW, Chi I. Cost-benefit analysis of a case management project for the community-dwelling frail elderly in Hong Kong. J Appl Gerontol. 2004;23(1):70-85.

6. Bucknall C, Miller G, Lloyd S. Glasgow supported self-management trial (GSuST) for patients with moderate to severe COPD: randomised controlled trial. BMJ. 2012;1060(March):1-13.

7. Trappenburg JC, Monninkhof EM, Bourbeau J, et al. Effect of an action plan with ongoing support by a case manager on exacerbation-related outcome in patients with COPD: a multicentre randomised controlled trial. Thorax. 2011;66(11):977-984.

8. Bourbeau J, Julien M, Maltais F, et al. Reduction of hospital utilization in patients with chronic obstructive pulmonary disease. Arch Intern Med. 2003;163:585-591.

9. Rice KL, Dewan N, Bloomfield HE, et al. Disease management program for chronic obstructive pulmonary disease: a randomized controlled trial. Am J Respir Crit Care Med. 2010;182(7):890-896.

10. Fan VS, Gaziano JM, Lew R, Bourbeau J, Adams SG. A comprehensive care management program to prevent chronic obstructive pulmonary disease hospitalizations: a randomized controlled trial. Ann Intern Med. 2012;156(10):673-683.

11. Koff PB, Jones RH, Cashman JM, Voelkel NF, Vandivier RW. Proactive integrated care improves quality of life in patients with COPD. Eur Respir J. 2009;33(5):1031-1038.

12. Moullec G, Lavoie KL, Rabhi K, Julien M, Favreau H, Labrecque M. Effect of an integrated care programme on re-hospitalization of patients with chronic obstructive pulmonary disease. Respirology. 2012;17(4): 707-714.

13. Bird S, Noronha M, Sinnott H. An integrated care facilitation model improves quality of life and reduces use of hospital resources by patients with chronic obstructive pulmonary disease and chronic heart failure. Aust J Prim Health. 2010;16(4):326-333.

14. Poole PJ, Chase B, Frankel A, Black PN. Case management may reduce length of hospital stay in patients with recurrent admissions for chronic obstructive pulmonary disease. Respirology. 2001;6(1):37-42. 
15. Long MJ, Marshall BS. What price an additional day of life? A costeffectiveness study of case management. Am J Manag Care. 2000;6(8): 881-886.

16. Newcomer R, Maravilla V, Faculjak P, Graves MT. Outcomes of preventive case management among high-risk elderly in three medical groups: a randomized clinical trial. Eval Health Prof. 2004;27(4): 323-348.

17. Efraimsson EO, Hillervik C, Ehrenberg A. Effects of COPD self-care management education at a nurse-led primary health care clinic. Scand J Caring Sci. 2008;22(2):178-185.

18. Bourbeau J, Collet J-P, Schwartzman K, Ducruet T, Nault D, Bradley C. Economic benefits of self-management education in COPD. Chest. 2006;130(6):1704-1711.

19. Sculpher MJ, Pang FS, Manca A, et al. Generalisability in economic evaluation studies in healthcare: a review and case studies. Health Technol Assess (Rockv). 2004;8(49):1-192.

20. Husereau D, Drummond M, Petrou S, et al. Consolidated Health Economic Evaluation Reporting Standards (CHEERS) - explanation and elaboration: a report of the ISPOR Health Economic Evaluation Publication Guidelines Good Reporting Practices Task Force. Value Heal. 2013;16:231-250.

21. Ramsey S, Willke R, Briggs A, et al. Good research practices for costeffectiveness analysis alongside clinical trials: the ISPOR RCT-CEA task force report. Value Heal. 2005;8(5):521-533.

22. Hulley SB, Cummings SR, Browner WS, Grady DG, Newman TB. Designing Clinical Research. 3rd ed. Philadelphia, PA: Lippincott Williams \& Wilkins; 2007.
23. Region Midtjylland. Det Nationale Indikatorprojekt. Kronisk Obstruktiv Lungesygdom (KOL). Standarder, indikatorer og prognostiske faktorer. 2007:45. Available from: http://www.regionmidtjylland.dk/. Accessed January 15, 2015.

24. Wittrup-Jensen KU, Lauridsen J, Gudex C, Pedersen KM. Generation of a Danish TTO value set for EQ-5D health states. Scand J Public Health. 2009;37(5):459-466.

25. Drummond MF, Sculpher MJ, Torrance GW, O'Brien BJ, Stoddart GL. Methods for the Economic Evaluation of Health Care Programmes. 3rd ed. New York: Oxford University Press; 2005.

26. Zwarentein M, Treweek S, Gagnier JJ, et al. Improving the reporting of pragmatic trials: An extension of the CONSORT statement. BMJ. 2008;337(a2390). doi:10.1136/bmj.a2390.

27. Moher D, Hopewell S, Schulz KF, et al. CONSORT 2010 explanation and elaboration: updated guidelines for reporting parallel group randomised trials. BMJ. 2010;340:c869.

28. Schafer JL. Multiple imputation: a primer. Stat Methods Med Res. 1999;8:3-15.

29. Gray AM, Clarke PM, Wolstenholme JL, Wordsworth S. Applied Methods of Cost-effectiveness Analysis in Health Care. 1st ed. New York: Oxford University Press; 2011.

30. Sullivan SD, Ramsey SD, Lee TA. The Economic Burden of COPD. Chest. 2000;117:5S-9S
Open Access Journal of Clinical Trials

\section{Publish your work in this journal}

The Open Access Journal of Clinical Trials is an international, peerreviewed, open access journal publishing original research, reports, editorials, reviews and commentaries on all aspects of clinical trial design, management, legal, ethical and regulatory issues, case record form design, data collection, quality assurance and data auditing

\section{Dovepress}

methodologies. The manuscript management system is completely online and includes a very quick and fair peer-review system, which is all easy to use. Visit http://www.dovepress.com/testimonials.php to read real quotes from published authors. 